\title{
Effects of Cisapride on Constipation Due to a Neurological Lesion
}

\author{
G. H. de Groot, $M D,{ }^{1}$ G. F. de Pagter, $M^{2}$ \\ Department of Internal Medicine ${ }^{1}$ and Urology ${ }^{2}$, St fozef Hospital, Mozartstraat \\ 90, 1962 AB Heemskerk, The Netherlands.
}

\section{Summary}

Two patients with intractable constipation and an atonic bladder due to a partial spinal cord lesion and sacral nerve lesion are described.

Treatment with cisapride $(4 \times 10 \mathrm{mg}$ daily) was undertaken. After a few days the stool passed spontaneously. The effect was dose-dependent and has been maintained for at least 40 months. Normal bladder function was not achieved.

Key words: Spinal cord lesion; Atonic colon; Neuropathic bladder; Cisapride.

\section{Introduction}

Faecal stasis with colonic dilation and urinary incontinence is a distressing problem in patients with a spinal cord lesion and iatrogenic neurological damage after lower pelvic surgery (Glick et al., 1984). Cholinergic agents have been used in an attempt to overcome the lack of para-sympathetic cholinergic stimulation but they are disappointing because of their side effects. Recently, cisapride has been described as a non-cholinergic gastro-intestinal motor stimulant Reyntjes et al. (1984), supposedly acting through increased release of acetylcholine in the intramural plexuses. Its prokinetic effects on gastro-intestinal motility have been demonstrated in patients with motility disorders of the upper gastro-intestinal tract (Jian, 1985; Smout et al., 1985), however its effect on colonic dysfunction with faecal stasis caused by lesions of the spinal cord or iatrogenic damage of the sacral nerves, has not been described. Recently, we have succesfully used cisapride in 2 patients with constipation of neurological constipation.

\section{Case 1}

A 38 year old man presented with a 14 year old history of constipation, which began in 1970 when he developed a slowly progressive spastic paresis of the lower extremities, and neuropathic urinary bladder disturbances.

Neurological investigations showed an atrophy of the spinal cord beyond the level

Correspondence to: Dr G. H. de Groot, St Jozef Hospital, Mozartstraat 90, 1962 AB Heemskerk, The Netherlands. 
of thoracic 5, and the CT-scan was confirmatory. Bowel movements did not occur spontaneously despite the use of the bulk laxative agents (hydrophilic agents, Senna and Lactulose). Manual evacuation of the bowel was ultimately the only effective way for bowel evacuation. Colonoscopic investigation showed a large bowel without peristaltic waves. Biopsies from the rectal mucosa showed ganglion cells and there were no signs of melanosis coli or amyloidosis. Haematological and biochemical examination did not show evidence for a systemic disorder or of diabetes mellitus.

It was concluded that the constipation was due to the spinal cord lesion.

The patient was treated with cisapride, $4 \times 10 \mathrm{mg}$ daily. From the third day onwards a stool was passed spontaneously once a day. Two months later we tried to reduce the dose to $2 \times 10 \mathrm{mg}$ daily, but this resulted in a relapse of the constipation. After restoring the original dose, bowel movement occurred spontaneously again once a day. After 3 months, discontinuation of the therapy for 1 week resulted in a prompt relapse, whereafter the cisapride treatment was reinstated with an excellent result. Resolution of constipation has been maintained now for more than 40 months. Transient side-effects included headache, nausea and increased frequency of micturition.

Cystometric measurements before the introduction of cisapride showed a lack of detrusor function. Cisapride therapy did not improve primary bladder function, but induced earlier and more spontaneous contractions of the bladder as shown by cystometry.

\section{Case 2}

A 50 year old man presented with a 16 year history of constipation. After lower anterior resection of rectum and sigmoïd colon in 1970 for carcinoma of sigmoïd (Dukes B), he developed impotence and severe constipation. Spontaneous bowel movement with previous sensation of defecation urge never occurred. Manual evacuation of the stool was necessary weekly.

Urological screening showed an atonic urinary bladder and colonoscopic examination demonstrated a dilated and atonic distal colon. There were no signs of mechanical obstruction by metastases.

It was concluded that the constipation and the atonic bladder dysfunction were due to iatrogenic damage of (sacral) recto-colon enervation. The patient was treated with cisapride $4 \times 10 \mathrm{mg}$ daily, and after 2 weeks the stool passed spontaneously once a day and the patient has regained the sensation of the urge to defecate.

\section{Discussion}

Cisapride is a gastro-intestinal prokinetic drug which has no anti-dopaminergic and no cholinergic effects. Its prokinetic effect on colon motility is not exactly understood but probably acts by stimulating directly the colonic smooth muscle and secondly by releasing acetylcholine at nerve endings in the myenteric plexus.

The propulsive effect of cisapride has been demonstrated in patients with chronic functional constipation (Müller-Lissner, 1986) and in patients with diabetic enteropathy (Rederer et al., 1986). It therefore seemed justified to treat our patients with cisapride. Although the results of our study are promising we are fully aware of the restrictions imposed by the open nature of the study and by having treated only two patients so far. However a placebo effect seems unlikely, because the improvement has been sustained now for more than 40 months, and furthermore the reduction of the dose led to relapse of the constipation.

Although cisapride did not result in normal bladder function, but increased 
contractability of the bladder, the ultimate effect resulted in more efficient bladder emptying with the absence of frequent bladder infection.

Our preliminary results justify further clinical trials of cisapride as a useful therapy of intractable constipation in patients with certain neural lesions.

\section{References}

Glick M, Meshrinpour H, Haldeman S et al., 1984. Colonic dysfunction in patients with thoracic spinal cord injury. Gastroenterology 86:287-294.

Jian R, DuCrot F, Piepeloup C et al., 1985. Measurements of gastric emptying in dyspeptic patients: effect of a new gastrokinetic agent (Cisapride). Gut 25:325-358.

MÜLLER-LISSNER S and the Bavarian Constipation Study Group, 1986. Cisapride in chronic constipation and laxative abuse. Digestion 34(2):158 (abstr.)

ReDerer PC, Ellermann A, Schmidt H et al., 1986. Effects of Cisapride on sigmoïd motility in healthy subjects and in diabetic enteropathy with constipation. Digestion 34(2):155 (abstr.)

ReYNTJES A, Verlinden M, SchuURKes J et al., 1984. New approach to gastro-intestinal motor dysfunction: non-antidopaminergic, non-cholinergic stimulation with Cisapride. Curr. Ther. Res. 36: 1029-1037.

Smout AJPM, BogaARD JW, Grade AC et al., 1985. Effects of Cisapride, a new gastro-intestinal prokinetic substance, on interdigestive and postprandial motor activity of the distal oesophagus in man. Gut 26: 246-259. 